Case Report

\title{
Leclercia adecarboxylata Musculoskeletal Infection in an Immune Competent Pediatric Patient: An Emerging Pathogen?
}

\author{
W. Jeffrey Grantham, ${ }^{1}$ Shawn S. Funk, ${ }^{1}$ and Jonathan G. Schoenecker ${ }^{2}$ \\ ${ }^{1}$ Vanderbilt University Medical Center, MCE South Tower, Suite 4200, 1215 21st Avenue South, Nashville, \\ TN 37232-8774, USA \\ ${ }^{2}$ Vanderbilt University Medical Center, 2200 Children's Way No. 11130, Nashville, TN 37232, USA \\ Correspondence should be addressed to Jonathan G. Schoenecker; jon.schoenecker@vanderbilt.edu
}

Received 12 July 2015; Accepted 28 September 2015

Academic Editor: Christian W. Müller

Copyright (c) $2015 \mathrm{~W}$. Jeffrey Grantham et al. This is an open access article distributed under the Creative Commons Attribution License, which permits unrestricted use, distribution, and reproduction in any medium, provided the original work is properly cited.

Case. An immune competent pediatric patient presented with a persistent lower extremity infection with Leclercia adecarboxylata after a penetrating injury. This case report details the presentation, clinical course, and treatment. Conclusion. Leclercia adecarboxylata has increasing reports in immunosuppressed and adult patients with musculoskeletal infection. This case now indicates that Leclercia adecarboxylata is a potential pathogen in immune competent children in musculoskeletal tissue.

\section{Introduction}

As a member of the Enterobacteriaceae family, Leclercia adecarboxylata is a motile Gram negative rod. First described in 1962, its description in the literature is isolated to case reports $[1,2]$. Leclercia adecarboxylata is regarded as normal gut flora for animals and has been isolated in human stool. The bacterium is primarily reported in immunosuppressed patients or polymicrobial infections [3]. There have been no case reports of Leclercia adecarboxylata in an immunocompetent musculoskeletal infection in a child. We present a case of foot infection associated with a foreign body by Leclercia adecarboxylata in an immunocompetent pediatric patient.

\section{Case Report}

A 9-year-old girl stepped on debris in a corn field while playing near a lake. She noted a penetrating injury to the left foot and presented to outside hospital where a foreign body was removed and was started on antibiotics. Foot pain persisted without systemic symptoms. Four days after the injury, pain persisted and she was taken to the operating suite for formal irrigation and debridement by an orthopaedic surgeon. The procedure removed a more substantial foreign body and a wick was placed in the wound. She completed three separate courses of antibiotics including cefalexin, sulfamethoxazole/trimethoprim, and cefepime for persistent drainage.

The patient presented to our institution two and one-half months after initial injury with continued pain and a small abscess at the medial midfoot. She was able to walk without a significant limp. Radiographs were negative for evidence of traumatic injury or foreign body (Figure 1(a)); however, MRI of the left foot had revealed a fluid collection surrounding a foreign body (Figures 1(b)-1(d)). Her laboratory markers were C-reactive protein $0.4 \mathrm{mg} / \mathrm{L}$ (normal 0.1-1.0 $\mathrm{mg} / \mathrm{L}$ ), erythrocytesedimentation rate $6 \mathrm{~mm} / \mathrm{hr}$ (normal $0-20 \mathrm{~mm} / \mathrm{hr}$ ), and white blood cell count 6,800 cells/mcL (normal 4,00013,200 cells/mcL).

She was taken to the operating room for irrigation and debridement. Aspiration was attempted with no significant purulence noted. The wound was explored and a $15 \times 3 \mathrm{~mm}$ foreign body was removed (Figure 2(a)). The wound was irrigated, debrided, and closed primarily. Wound cultures were sent intraoperatively. The only organism isolated on blood and MacConkey agars was Leclercia adecarboxylata 


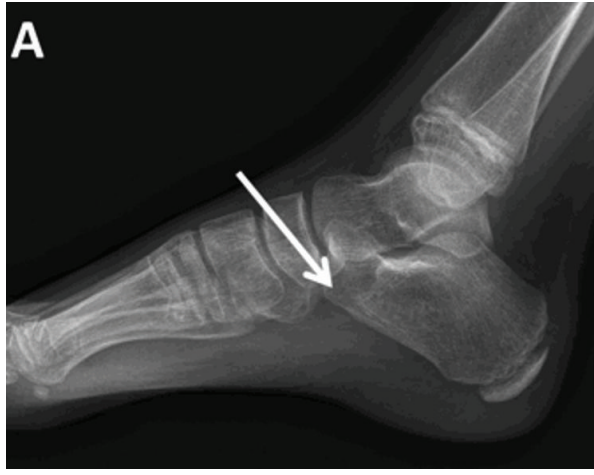

(a)

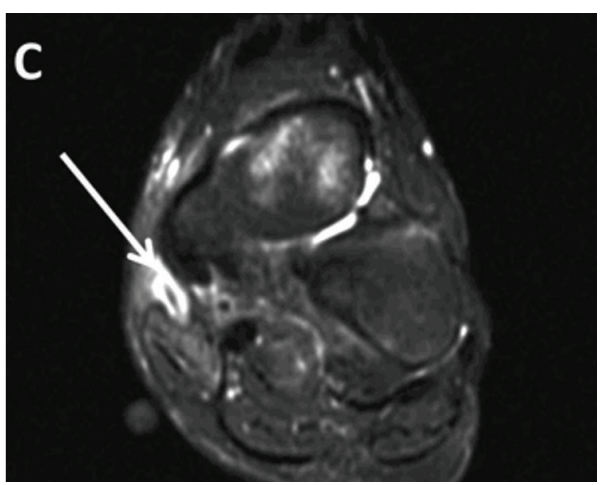

(c)

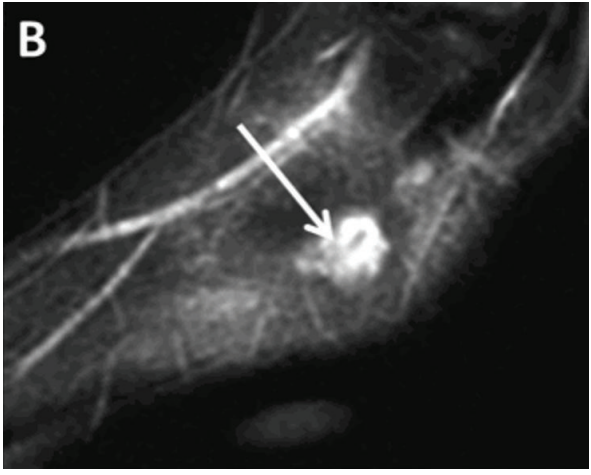

(b)

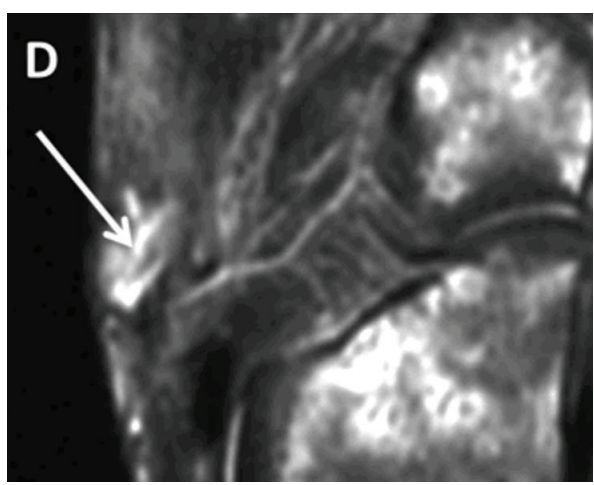

(d)

FIGURE 1: (a) X-ray and ((b)-(d)) sagittal, axial, and coronal MRI, respectively, indicating abscess with potential foreign body.

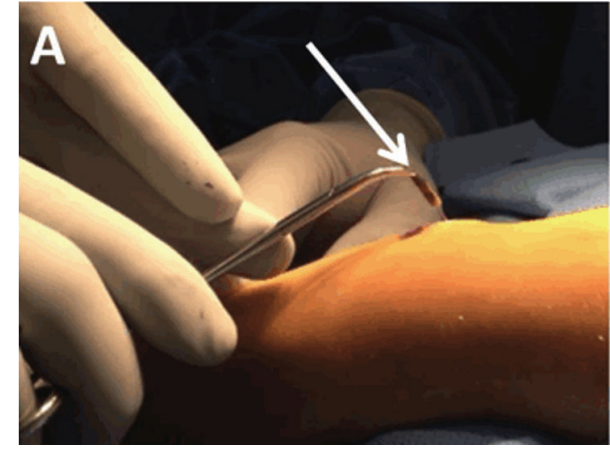

(a)

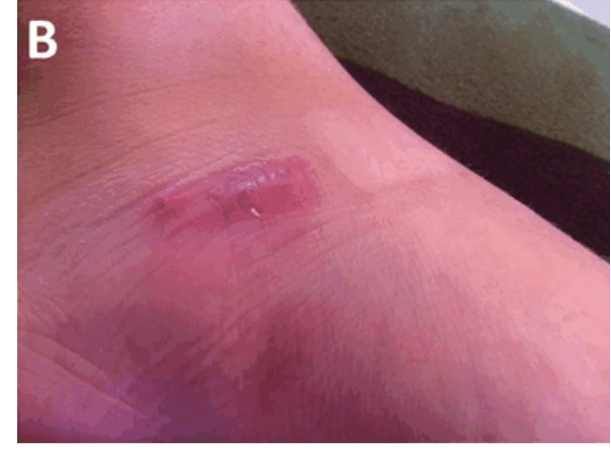

(b)

FiguRE 2: (a) Foreign body removal and (b) two-month follow-up.

with susceptibility to multiple antibiotics and resistance to ampicillin. After consultation with pediatric infectious disease the patient was treated with a 14-day course of levofloxacin. Two months postoperatively there were no signs of continued infection or wound complications (Figure 2(b)). One year following her injury, she is without complaints.

\section{Discussion}

This case presents the first time Leclercia adecarboxylata has been isolated in an immunocompetent pediatric patient.
Prior reports document this bacterium in immunocompromised patients [3-8]; however, recently there have been limited reports in immunocompetent patients $[9,10]$ in addition to the pediatric population $[5,8,11]$. Most cases report the bacteria with excellent susceptibility to antibiotics, as was the case for this isolate; however, resistant strains have been found [12]. Even catheter-associated infection with Leclercia adecarboxylata has been treated successfully with antibiotics alone without catheter removal [13]. In our case, the retained foreign body provided a medium for the persistent indolent infection despite courses of three different antibiotics. With removal of the foreign body the persistent infection resolved 
with routine oral antibiotic course. This bacterium, which previously had minimal recognition, now has increasing reports and is associated with pediatric immunocompetent patients with musculoskeletal infection. This report indicates that Leclercia adecarboxylata is a potential pathogen in immunocompetent children in musculoskeletal tissue.

\section{Conflict of Interests}

The authors declare that there is no conflict of interests regarding the publication of this paper.

\section{References}

[1] H. Leclerc, "Biochemical study of pigmented Enterobacteriaceae," Annales de l'Institut Pasteur, vol. 102, pp. 726-741, 1962.

[2] Z. Temesgen, D. R. Toal, and F. R. Cockerill III, "Leclercia adecarboxylata infections: case report and review," Clinical Infectious Diseases, vol. 25, no. 1, pp. 79-81, 1997.

[3] R. M. Daza, J. Iborra, N. Alonso, I. Vera, F. Portero, and P. Mendaza, "Isolation of Leclercia adecarboxylata in a cirrhotic patient," Enfermedades Infecciosas y Microbiologia Clinica, vol. 11, no. 1, pp. 53-54, 1993.

[4] M. M. Martinez, G. Sanchez, J. Gomez, P. Mendaza, and R. M. Daza, "Isolation of Leclercia adecarboxylata in ulcer exudate," Enfermedades Infecciosas y Microbiología Clínica, vol. 16, no. 7, p. $345,1998$.

[5] O. Fattal and J. G. Deville, "Leclercia adecarboxylata peritonitis in a child receiving chronic peritoneal dialysis," Pediatric Nephrology, vol. 15, no. 3-4, pp. 186-187, 2000.

[6] J. A. Rodríguez, F. J. Sánchez, N. Gutiérrez, J. E. García, and J. A. García-Rodríguez, "Bacterial peritonitis due to Leclercia adecarboxylata in a patient undergoing peritoneal dialysis," Enfermedades Infecciosas y Microbiologia Clinica, vol. 19, no. 5, pp. 237-238, 2001.

[7] A. Beltrán, A. V. Molinero, S. Capilla, and A. M. Polo, "Isolation of Leclercia adecarboxylata from wound exudate of a diabetic patient," Medicina Clinica, vol. 122, no. 4, article 159, 2004.

[8] A. Shah, J. Nguyen, L. M. Sullivan, K. R. Chikwava, A. C. Yan, and J. R. Treat, "Leclercia Adecarboxylata cellulitis in a child with acute lymphoblastic leukemia," Pediatric Dermatology, vol. 28, no. 2, pp. 162-164, 2011.

[9] P. Davenport and K. J. Land, "Isolation of Leclercia adecarboxylata from the blood culture of an asymptomatic platelet donor," Transfusion, vol. 47, no. 10, pp. 1816-1819, 2007.

[10] B. Hess, A. Burchett, and M. K. Huntington, "Leclercia adecarboxylata in an immunocompetent patient," Journal of Medical Microbiology, vol. 57, no. 7, pp. 896-898, 2008.

[11] K. A. Myers, R. M. Jeffery, and A. Lodha, "Late-onset Leclercia adecarboxylata bacteraemia in a premature infant in the NICU," Acta Paediatrica, vol. 101, no. 1, pp. e37-e39, 2012.

[12] E. H. Eiland, H. Siddiqui, A. M. Goode, and S. D. Leeth, "Pneumonia due to multidrug-resistant Leclercia adecarboxylata," American Journal of Health-System Pharmacy, vol. 70, no. 11, pp. 940-941, 2013.

[13] M. Fernández-Ruiz, F. López-Medrano, L. García-Sánchez et al., "Successful management of tunneled hemodialysis catheterrelated bacteremia by Leclercia adecarboxylata without catheter removal: report of two cases," International Journal of Infectious Diseases, vol. 13, no. 6, pp. e517-e518, 2009. 


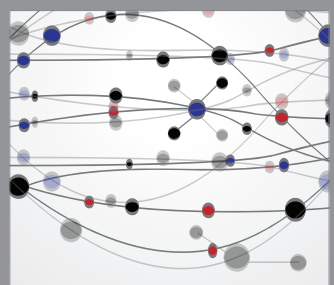

The Scientific World Journal
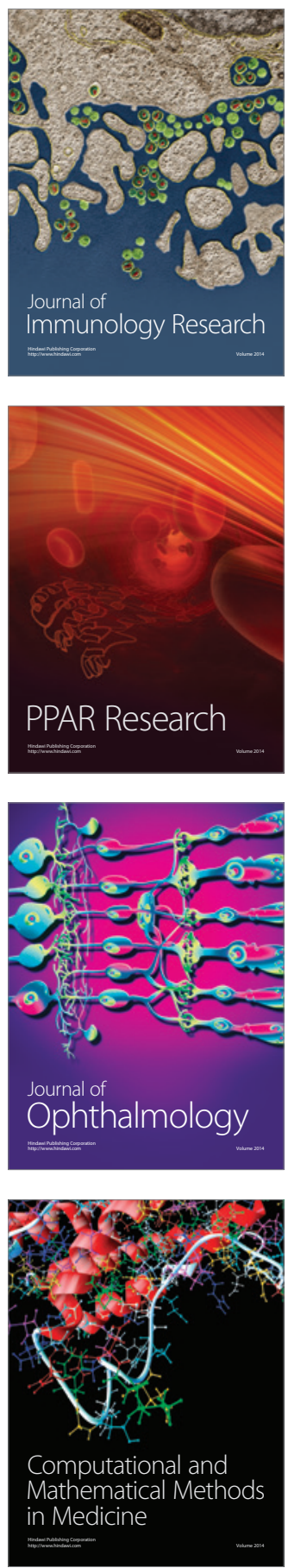

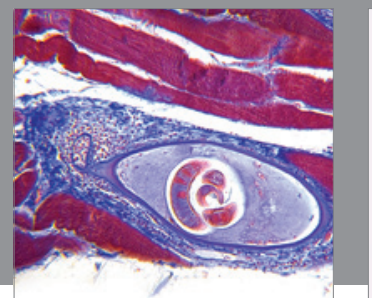

Gastroenterology

Research and Practice
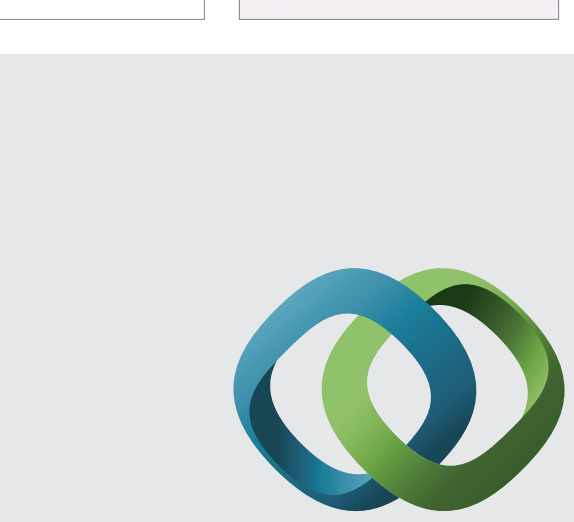

\section{Hindawi}

Submit your manuscripts at

http://www.hindawi.com
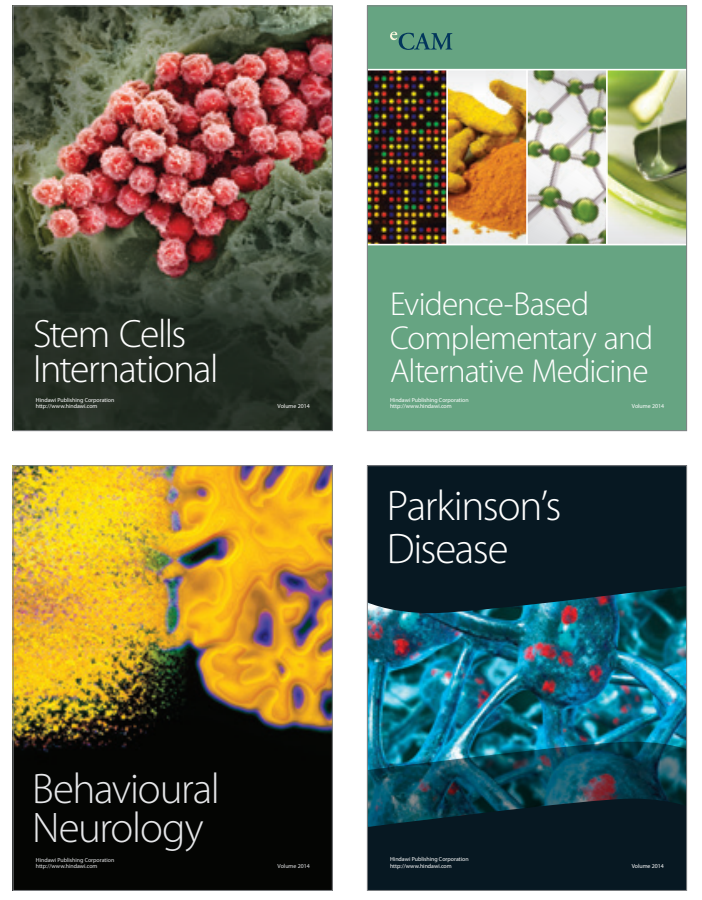
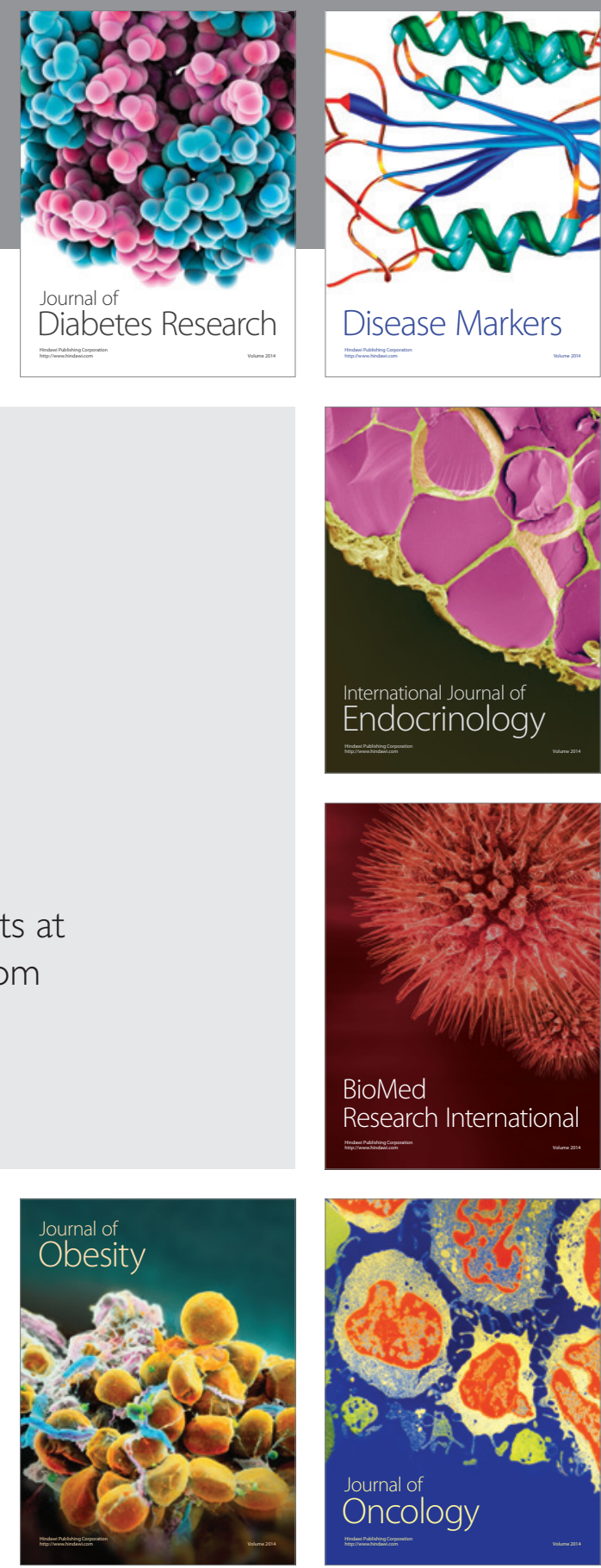

Disease Markers
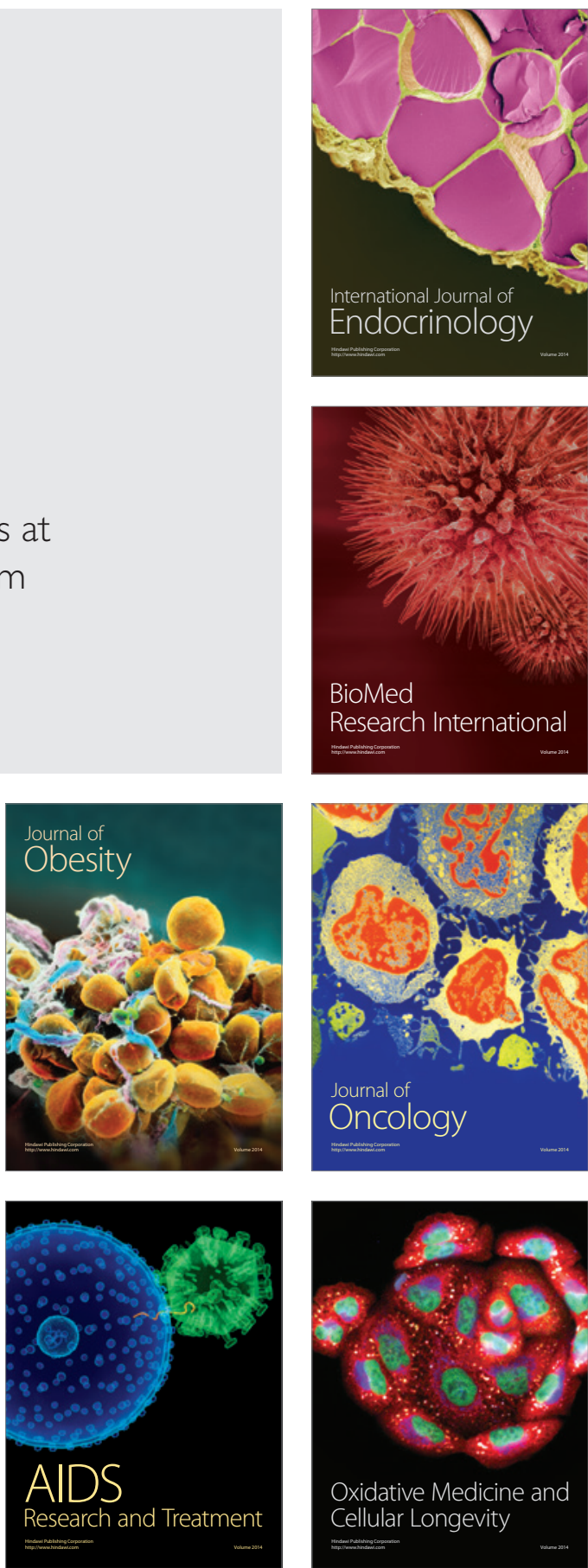